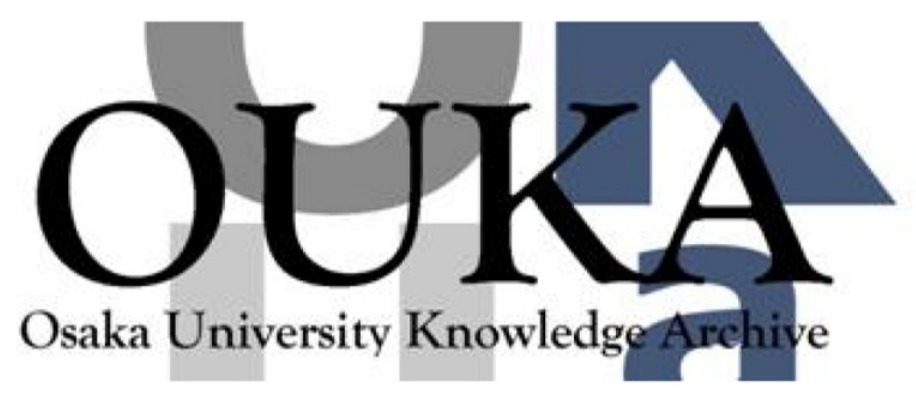

\begin{tabular}{|c|l|}
\hline Title & $\begin{array}{l}\text { Role of Vortical Structures on the Forced } \\
\text { Convective Heat Transfer in Oscillation- } \\
\text { Controlled Coaxial-Pipe Heat Exchanger }\end{array}$ \\
\hline Author(s) & $\begin{array}{l}\text { Takagi, Daisuke; Takeuchi, Shintaro; Kajishima, } \\
\text { Takeo }\end{array}$ \\
\hline Citation & $\begin{array}{l}\text { Journal of Enhanced Heat Transfer. 22(5) p. 365- } \\
\text { p.389 }\end{array}$ \\
\hline Issue Date & 2015 \\
\hline oaire:version & AM \\
\hline URL & https://hdl.handle.net/11094/57201 \\
\hline rights & \\
\hline Note & \\
\hline
\end{tabular}

Osaka University Knowledge Archive : OUKA

https://ir. Library. osaka-u. ac. jp/

Osaka University 


\title{
Role of Vortical Structures on the Forced Convective Heat Transfer in Oscillation-Controlled Coaxial-Pipe Heat Exchanger
}

\author{
Daisuke Takagi ${ }^{1}$, Shintaro Takeuchi ${ }^{1}$ and Takeo Kajishima ${ }^{1}$ \\ ${ }^{1}$ Department of Mechanical Engineering, Osaka University \\ 2-1 Yamada-oka, Suita-city, Osaka 565-0871 Japan
}

\begin{abstract}
A numerical simulation of an oscillation-controlled heat-transport coaxial pipe is carried out for studying the flow structure and heat transport characteristics. The heat-transport pipe connects the hot and cold reservoirs, and the cold and hot fluids are discharged with the anti-phase reciprocal waves through circular and annular openings at the ends of the chamber, respectively. By changing the diameter ratio of the circular opening to the inner tube, a unidirectionally circulating flow is observed to develop spontaneously. The flow rate of the unidirectional current is found to be approximately inversely-proportional to the diameter ratio. While the amount of transported heat increases with the flow rate of the unidirectional current (especially when generating strong vortices at the edge of the inner tube) for both cases of thermally-insulated and fully-conductive inner wall, the optimal heat transport performance is attained (not in association with the strongest unidirectional flow) when making zero-averaged vorticity in the cold-end region. For the case of the conductive inner wall, the heat loss across the wall is suppressed with decreasing the diameter ratio, due to the development of the strong jet and radially surrounding shear layer that separate the outer hot fluid from the inner cold fluid.
\end{abstract}

keywords: Active technique; Single-phase flow convection; Double-pipe heat exchanger; Computational modelling 


\section{Introduction}

Heat and mass transfer device is one of the crucial components for the systems in mechanical, geophysical and aeronautical areas, and great efforts have been paid for achieving higher efficiency. The efficient systems take advantage of the convective and diffusive transfers of heat and mass with simple mechanism. A good example is a transport system by an externally-induced reciprocal flow in a pipe, also known as dream-pipe [1]. In the device, heat is transported by longitudinal convection and lateral diffusion in a liquid subjected to periodic oscillation through a single-layered pipe under a longitudinal temperature gradient. Given a tuned frequency, it is reported that a high heat-transport performance far better than heat conduction through copper was attained with no net transfer of mass [1]. Also for biological systems, heat and mass transfer is of vital importance to maintain metabolism. The idea of the above longitudinal transport by external oscillation in a pipe originally developed for predicting the oxygen dispersion in pulmonary systems $[2,3,4,5]$. Meanwhile, an interesting gas exchange mechanism is known in the respiratory system of birds [6] and certain families of reptiles [7, 8]; during inhalation and exhalation, air can flow through the looped structure of bronchi and lungs continuously, by expanding and contracting the air sacs. Then, a unidirectional flow (with oscillating component) of gas develops in the air path along the looped structure. Although two full respiratory cycles are needed for a single-inhaled volume of gas to complete its path [6], the spontaneous gas circulation develops without mechanical valves, which facilitates the gas exchange for birds in high altitudes. Wang et al.[9] experimentally studied a model of birds respiratory organs and mentioned that a formation of a confined jet in the wake of the constriction placed just upstream of the bifurcation of the main tube (a model of a bronchi) could increase the convective inertia, giving rise a unidirectional current. Sakai et al. [10] experimentally showed that the vortex is effectively generated by the constriction, and that a separation vortex near the bifurcation of the tubes plays a vital role. They also proposed a mass transport model in the bifurcated pipe system [10].

Ishii et al. [11] introduced a novel oscillation-controlled transport device featuring coaxial doublelayered pipes connecting the two reservoirs through circular and annular openings at the respective ends. This simple structure has, literally, the same topology as the respiratory system of birds; given periodic oscillations to the fluids at the circular and annular openings with a certain phase difference, apparent steady flows develop superposed with reciprocal flow components in both inner (circular) and outer (annular) conduits [11]. The apparent steady flows are in the opposite directions to each other in the conduits, making a unidirectional current, as if circulating from the inner tube to the annulus and back to the inner tube again. 


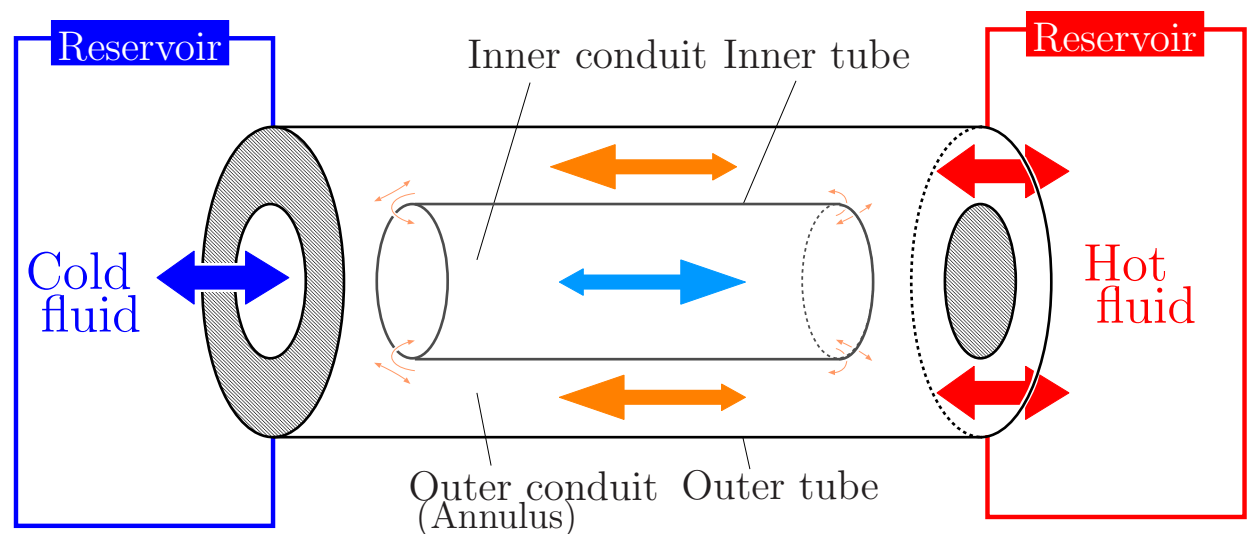

Figure 1: Schematic of oscillation-controlled coaxial heat-transport pipe.

Further, Ishii et al. [11] applied it to a heat exchanger by connecting the both ends of the double-tube structure to the cold and hot reservoirs, as shown in Fig. 1. They studied the effects of the oscillating frequency and amplitude on the amount of heat transported in the coaxial double-tube structure. Hishida et al. [12] reported that the coaxial-pipe system shows better heat transport rate (by $10 \sim 490$ times) than that by the oscillation-controlled single-layered pipe based on the theoretical model of Kaviany [13]. Although the dramatic enhancement in heat transport rate is caused by the unidirectional-circulating current between two reservoirs, their axisymmetric numerical simulation also shows a tendency that larger frequency and tidal amplitude cause a larger fraction of heat transported by the reciprocal flow component [12].

For both single-layered structure and coaxial double-tube system, loss of the performance is caused by an asynchronised flow or heat flux. Inaba et al. [14] showed that, at sufficiently high frequency in a single-layered pipe, there is a region near the pipe wall where the heat is transferred from the cold to high temperature sides due to a large phase difference between the temporal changes in fluid motion and temperature. This problem could be avoided and improved by adjusting the frequency to the characteristic time scale of the system. Nishio et al. [15] showed that the heat exchange was effectively enhanced for bundled conductive single-layered pipes by oscillating the fluids in the neighbouring channels at the anti-phase. However, this measure does not apply to the double-tubed chamber, as the coaxial-tube system transports heat by a spontaneous unidirectional circulating current. Therefore, heat conduction across the inner wall has to be avoided, which is the opposite requirement to the bundled single-pipe structure. Meanwhile, particularly for the double-tube structure, another reason for deterioration of the efficiency is generation of vortices at the bends and in the tubes. Considering the studies on the birds lung models in the above, the amount of fluid and heat transported in an oscillation-controlled coaxial pipe could increase by a formation of a strong stream that persists downstream in the inner tube; however, at the same time, the mechanical work also increases to push/drain the fluid into/out of the small pipe. Despite the above studies from the experimental and numerical aspects, the mechanisms that influence the heat-transfer rate of the coaxial heat 
exchanger have not been fully studied. Particularly, the transport mechanism in association with the vortical structures needs more attention, and careful observation and analysis of the vortical structures would help to work out with further efficient mechanism and to understand the biological functions.

In the present study, heat and mass transfer in the oscillation-controlled coaxial heat-transport pipe is numerically studied by focusing on the flow structure. We study the heat transport characteristics by focusing on the unidirectional-circulating flow developing in the inner and outer conduits. Also, characteristic vortical structures are observed to be relevant to entraining a fluid from annulus to inner tube, to facilitating smooth return flow towards the exit and to a possible improvement of the transport efficiency. By comparing the cases of the insulated inner wall (i.e., the ideal situation) and conductive wall, the isolated effect of the heat transfer across the inner tube wall is investigated, and a predominant factor that affects the heat transport even after a strong mixing by the passage of an emerging jet is discussed.

\section{Governing equations and computational setup}

\subsection{Governing equations and numerical methods}

Incompressible Newtonian fluid is used, and the density, viscosity and heat conductivity of the fluid are assumed to be constant. The governing equations are non-dimensionalised by the reference length $L$, the reference velocity $V$, the reference pressure $\rho U^{2}$ with the fluid density $\rho$ and the characteristic temperature difference $\Delta \Theta$, and the following non-dimensional numbers exist:

$$
\operatorname{Re}=\frac{V L}{\nu}, \quad \operatorname{Pr}=\frac{\nu}{\kappa},
$$

where the kinematic viscosity $\nu$ and the thermal conductivity $\kappa$. As the heat and fluid flows in circular and annular tubes are treated in this study, the continuity equation, Navier-Stokes equations and energy equation are described in the cylindrical coordinate system:

$$
\begin{aligned}
& \frac{1}{r} \frac{\partial r u_{r}}{\partial r}+\frac{1}{r} \frac{\partial u_{\theta}}{\partial \theta}+\frac{\partial u_{z}}{\partial z}=0, \\
& \frac{\partial u_{r}}{\partial t}+\Xi u_{r}-\frac{u_{\theta}^{2}}{r}=-\frac{\partial p}{\partial r}+\frac{1}{\operatorname{Re}}\left(\Lambda u_{r}-\frac{u_{r}}{r^{2}}-\frac{2}{r^{2}} \frac{\partial u_{\theta}}{\partial \theta}\right), \\
& \frac{\partial u_{\theta}}{\partial t}+\Xi u_{\theta}+\frac{u_{r} u_{\theta}}{r}=-\frac{\partial p}{\partial \theta}+\frac{1}{\operatorname{Re}}\left(\Lambda u_{\theta}-\frac{u_{\theta}}{r^{2}}+\frac{2}{r^{2}} \frac{\partial u_{r}}{\partial \theta}\right), \\
& \frac{\partial u_{z}}{\partial t}+\Xi u_{z}=-\frac{\partial p}{\partial z}+\frac{1}{\operatorname{Re}} \Lambda u_{z}, \\
& \frac{\partial \Theta}{\partial t}+\Xi \Theta=\frac{1}{\operatorname{Re} \cdot \operatorname{Pr}} \Lambda \Theta,
\end{aligned}
$$




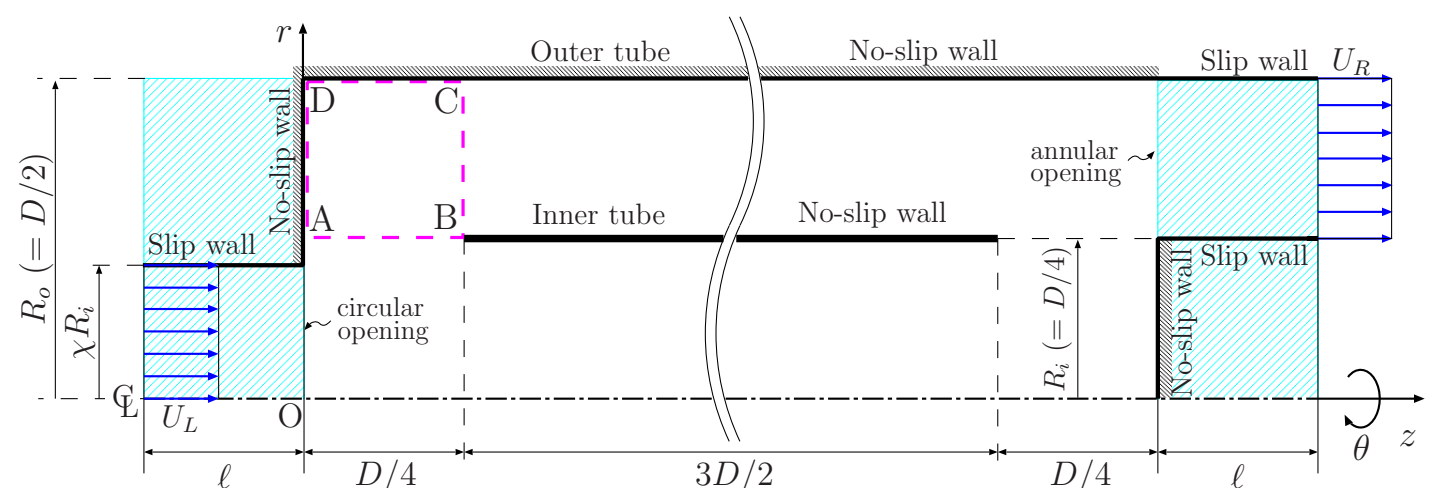

Figure 2: Computational domain and boundary conditions for the fluid velocities, and the region $\mathrm{ABCD}$ for the integral Eq.(6).

where, $r, \theta, z$ are the radial, circumferential and axial coordinates, respectively, $u_{r}, u_{\theta}, u_{z}$ are the velocity components in the respective directions, $p$ the pressure and $\Theta$ is the temperature. The differential operators $\Xi$ and $\Lambda$ are given as follows:

$$
\begin{aligned}
& \Xi=u_{r} \frac{\partial}{\partial r}+\frac{u_{\theta}}{r} \frac{\partial}{\partial \theta}+u_{z} \frac{\partial}{\partial z} \\
& \Lambda=\frac{1}{r} \frac{\partial}{\partial r}\left(r \frac{\partial}{\partial r}\right)+\frac{1}{r^{2}} \frac{\partial^{2}}{\partial \theta^{2}}+\frac{\partial^{2}}{\partial z^{2}}
\end{aligned}
$$

In the present study, external force, such as gravity, is not considered, and viscous dissipation is assumed to be negligible.

Although the results presented in this paper are pseudo three dimensional, we employ a full 3-D cylindrical coordinate system for future study of three dimensional vortical structures for enhancing the heat transfer by controlling the flow. A central finite difference scheme of a 2nd order accuracy is employed, and the velocity-pressure coupling is performed by the SMAC method [16].

\subsection{Computational setup}

Oscillation-controlled heat transport device of the present study has a coaxial-tube structure that connects hot and cold reservoirs, as Fig. 1 shows. In the computational model of the device, the fluid is allowed to come into and go out of the domain (from/to the cold and hot reservoirs) through the circular and annular openings at the left and right ends of the chamber, respectively.

Figure 2 shows the computational domain. The thickness of the inner tube is set to be zero. With keeping the sizes of the inner tube and the annular opening, the diameter of the circular opening is varied. Hereafter, the ratio of the diameters of the circular opening and inner tube is represented by $\chi$. 
Table 1: Computational parameters

\begin{tabular}{c|c|c}
\hline Num. Grid points & $N_{z} \times N_{r} \times N_{\theta}$ & $160 \times 60 \times 64$ \\
\hline Time increment & $\Delta t$ & $1.0 \times 10^{-3}$ \\
\hline Reynolds number & $\operatorname{Re}$ & 500 \\
\hline Prandtl number & $\operatorname{Pr}$ & 1 \\
\hline Frequency (non-dim.) & $f$ & 0.5 \\
\hline Diameter ratio & $\chi$ & $1.0,0.9,0.8,0.7,0.6,0.5$ \\
\hline
\end{tabular}

Computational parameters are summarised in Tab. 1. Reynolds number is defined based on the diameter of the outer tube $D$, the reference velocity $U$ (explained later) and the kinematic viscosity $\nu$. The diameter ratio $\chi$ is varied in the range between 0.5 and 1 . Unless specified otherwise, the oscillation frequency $f$ is fixed to be 0.5 since in a preliminary study the flows in both inner and outer conduits are found to widely change (from weak to strong unidirectional flows) for different $\chi$ values employed, as will be detailed later. For the initial condition, stationary fluid and zero temperature are assumed in the whole domain.

Figure 2 also shows the boundary conditions. No-slip boundary condition is specified at the walls including the annular and circular walls at the left and right ends of the chamber. In order to naturally replicate the flow from/to the reservoirs, a sub-domain of length $\ell(=0.5 D)$ is placed at each end of the chamber, depicted as hatched regions in Fig. 2, and the slip wall condition is applied. At the left end of the sub-domain in the cold-reservoir side, a temporally-varying fluid velocity (of top-hat profile) $u_{L}$ of the following form is given:

$$
u_{L}(t)=\frac{1}{\chi^{2}} U \sin (2 \pi f t)
$$

where $t$ is time and $U$ is the maximum velocity for the case of $\chi=1$. Note that the coefficient $1 / \chi^{2}$ in Eq.(1) is to allow the same inflow rate at the circular opening regardless of the value of $\chi$ employed. On the other hand, on the right end of the sub-domain in the hot-reservoir side, the following boundary velocity $u_{R}$ is prescribed:

$$
u_{R}(t)=\frac{U}{3} \sin (2 \pi f t+\phi)
$$

where $\phi$ is the phase difference. The coefficient $1 / 3$ is obtained as the diameters of the annular openings are remain unchanged

$$
\frac{\pi R_{i}^{2}}{\pi R_{o}^{2}-\pi R_{i}^{2}}=\frac{1}{3}
$$

throughout the present simulations. 
As for the temperature boundary condition, the thermally-insulated walls are specified, including the inner tube unless specified otherwise. Assuming hot and cold reservoirs in the respective ends of the domain, the temperature boundary conditions at the circular and annular openings are switched depending on the incoming/outgoing phase of the fluid flow. At the circular opening on the left end of the domain $(z=0)$, the following boundary conditions for temperature $\Theta_{L}$ are specified:

$$
\begin{array}{ll}
\text { Inflow phase }[\sin (2 \pi f t) \geq 0]: & \Theta_{L}=0 \\
\text { Outflow phase }[\sin (2 \pi f t)<0]: & \frac{\partial \Theta_{L}}{\partial t}+U_{m L} \frac{\partial \Theta_{L}}{\partial z}=0,
\end{array}
$$

where $U_{m L}$ is the mean velocity of $u_{L}(t)$ at $z=0$ cross-section. The boundary condition for temperature $\Theta_{R}$ at the annular opening on the right end of the domain $(z=2 D)$ is also switched as follows:

$$
\begin{array}{ll}
\text { Inflow phase }[\sin (2 \pi f t)<0]: & \Theta_{R}=1 \\
\text { Outflow phase }[\sin (2 \pi f t) \geq 0]: & \frac{\partial \Theta_{R}}{\partial t}+U_{m R} \frac{\partial \Theta_{R}}{\partial z}=0,
\end{array}
$$

where $U_{m R}$ is the mean velocity of $u_{R}(t)$ at $z=2 D$ cross-section.

Note that the annular flow can be tuned to approximately synchronise with the passage of the jet from the circular opening, given the present dimensions of the device (Fig.2), the maximum inflow velocities $\left(U / \chi^{2}\right.$ and $U / 3$, see Eqs.(1)(2)), oscillation frequency $f=0.5$ (Tab.1) and the phase-lag of $\phi=\pi[\mathrm{rad}]$ for injection phases from the circular and annular openings. This is obtained as the strengthening condition for the annular flow (in the returning phase toward the colder end) with the jet injection at the left edge of the inner tube. Although this condition assumes that the wave front travels with no attenuation in the annulus, it is still useful for estimating the operating condition. In a similar way, for $m$-th and $n$-th $(m, n \geq 1)$ injections from the annular and circular openings, respectively, the anti-synchronised (i.e., weakening-interference) condition is obtained such that a half-integer period of the jet injection matches with a half-integer period delay $(\phi=\pi)$ plus the elapsed time for the returning flow in the annulus. This condition reads:

$$
\left(n+\frac{1}{2}\right) \frac{1}{f} \simeq\left(m-\frac{1}{2}\right) \frac{1}{f}+\frac{7 D / 4}{U / 3} .
$$

By giving some pairs of integers for $m$ and $n$, the required frequencies for the anti-synchronised operation are obtained as $f \simeq 0.4(m, n=1,2), 0.6(m, n=1,3) \cdots$. 


\subsection{Validation}

To study the transport of heat and mass under oscillating flow in the present study, time-response of the flow field is of importance. Therefore, the numerical solutions of the flows driven by a sinusoidally-varying pressure gradient in the coaxial cylindrical tubes are compared with the analytical solution in an infinitelylong coaxial tube.

The fluid is driven by the following pressure gradient in the axial direction:

$$
\frac{\partial p}{\partial z}=\frac{\mathrm{d} p_{0}}{\mathrm{~d} z} \cos (2 \pi f t)
$$

where $\mathrm{d} p_{0} / \mathrm{d} z$ is set to be constant.

Assuming that the flow is axisymmetric and $u_{r}=u_{\theta}=0$, the analytical solutions of the axial velocity in the circular and annular conduits $\left(u_{\text {circ }}(t)\right.$ and $\left.u_{\text {ann }}(t)\right)$ are given as follows:

$$
\begin{aligned}
& u_{\text {circ }}(r, t)=\operatorname{Real}\left[\frac{i}{\omega}\left(-\frac{\mathrm{d} p_{0}}{\mathrm{~d} z}\right)\left\{\frac{J_{0}(\alpha r)}{J_{0}\left(\alpha R_{i}\right)}-1\right\} e^{i \omega t}\right] \\
& u_{a n n}(r, t)=\operatorname{Real}\left[\frac{i}{\omega}\left(-\frac{\mathrm{d} p_{0}}{\mathrm{~d} z}\right)\left\{C_{J} J_{0}(\alpha r)-C_{Y} Y_{0}(\alpha r)-1\right\} e^{i \omega t}\right],
\end{aligned}
$$

where $i$ is the imaginary unit, $\omega=2 \pi f, \alpha=\sqrt{i^{3} \omega / \nu}, J_{0}$ and $Y_{0}$ are the 0 -th order Bessel functions of the first and second kinds, respectively. The coefficients $C_{J}$ and $C_{Y}$ are given as follows:

$$
\begin{aligned}
C_{J} & =\frac{Y_{0}\left(\alpha R_{o}\right)-Y_{0}\left(\alpha R_{i}\right)}{J_{0}\left(\alpha R_{i}\right) Y_{0}\left(\alpha R_{o}\right)-J_{0}\left(\alpha R_{o}\right) Y_{0}\left(\alpha R_{i}\right)}, \\
C_{Y} & =\frac{J_{0}\left(\alpha R_{o}\right)-J_{0}\left(\alpha R_{i}\right)}{J_{0}\left(\alpha R_{i}\right) Y_{0}\left(\alpha R_{o}\right)-J_{0}\left(\alpha R_{o}\right) Y_{0}\left(\alpha R_{i}\right)},
\end{aligned}
$$

where $R_{i}$ and $R_{o}$ are the radii of the inner and outer tubes, respectively. By adjusting to the present simulation condition, $R_{i}=D / 4$ and $R_{o}=D / 2$ are specified.

For the numerical solution of this validation problem, the inner and outer tubes are the same length, and no-slip and periodic boundary conditions are given at the tube walls and tube ends, respectively.

Figure 3 compares the axial velocity profiles of numerical and analytical solutions. The five profiles are taken consecutively at constant intervals after reaching quasi-steady state $t=t_{d}(=60)$ and represented with the phase angles normalised by one period of oscillation $T(=2 \pi / \omega)$. The figure shows good agreement between the numerical and analytical solutions. The relative errors evaluated by $L^{2}$-norms and time-average over one period of oscillation are found to be $5.85 \times 10^{-3}$ and $7.76 \times 10^{-3}$ in the inner and outer conduits, respectively. The above results certify the accuracy of the time-oscillating flow.

In addition, the transport phenomena in a turbulent round jet have been compared with the measurement elsewhere $[17,18]$. 


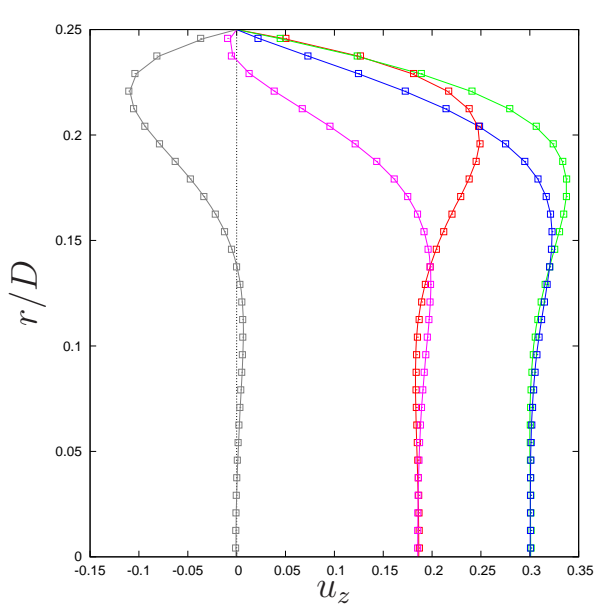

(a) Inner conduit
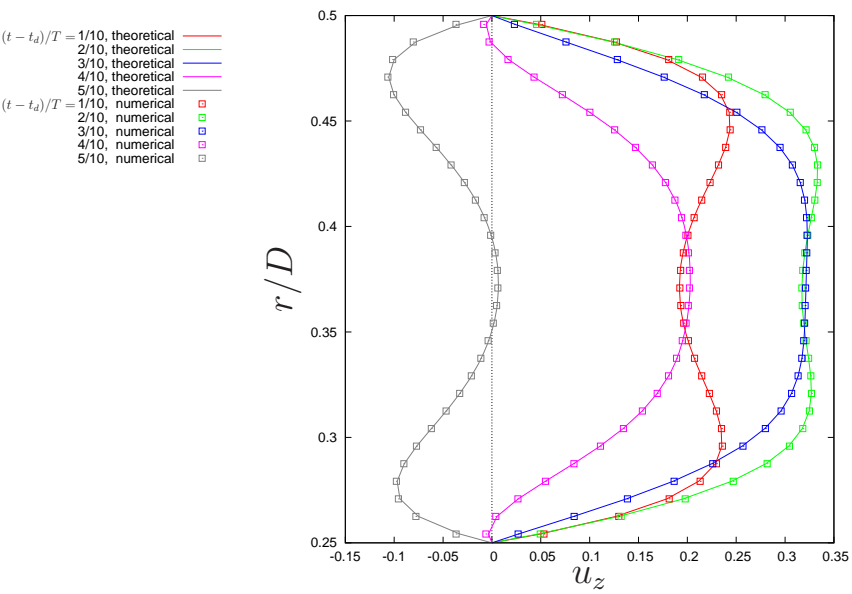

(b) Outer conduit

Figure 3: Comparison of the analytical and numerical profiles (lines and symbols, respectively) of the timedependent flows in the (a) inner circular conduit and (b) annulus. The numerical results are taken at constant intervals after reaching quasi-steady state $\left(t=60 \equiv t_{d}\right)$.

\section{Flow in the oscillation-controlled coaxial heat transport pipe}

Prior to the heat transport problem, we look into the development of the flow field, especially unidirectional flow, in association with the vortical structures.

\subsection{Development of the flow field}

Figures $4 \sim 6$ show typical behaviours of the flow and temperature fields for three cases of different diameter ratios, $\chi=1.0,0.7,0.5$, at constant intervals over one period $T(=1 / f)$ of oscillation from $t=t_{s}(=100 T)$, together with the radial profiles of the axial velocity $u_{z}$ in the cross-section at $z=D$ (the mid cross-section). Note that, as will be shown later, the flow and the temperature fields are regarded fully-developed by $t=5 T$ and $t=60 T$, respectively, for all the parameter cases. In the figures, the arrows and lines represent the velocity vectors (plotted at every three grid points) and temperature contours in the $r$ - $z$ cross-section. We employed complementary colours for representing the velocity vectors of a strong/weak stream (dark/bright colour) and the high/low temperature contour (bright/dark colour).

In the case of $\chi=1.0$ (Fig. 4), the fluid in the inner and outer conduits are found to change the flow direction in response to the oscillation at the tube ends, but the main flow directions in both conduits are approximately in-phase. At $t-t_{s}=T / 4$, a strong separation is formed near the left inlet, and it develops into a vortex by $t-t_{s}=T / 2$ when the inflow and outflow are switched. At $t-t_{s}=3 T / 4$, the return flow meanders from the annulus towards the left exit (cold-reservoir side) with a strong stream along the left-end wall, which reflects the effect of the counter-clockwise vortex (around $\theta$ axis) in this region. 


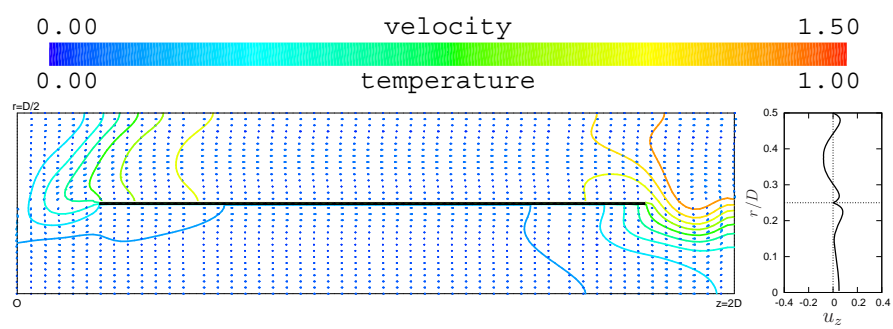

(a) $t-t_{s}=0$
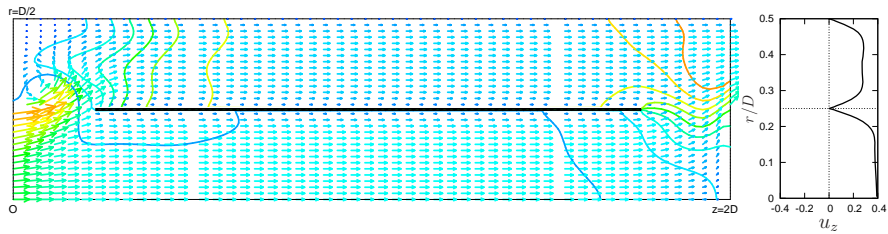

(b) $t-t_{s}=T / 4$
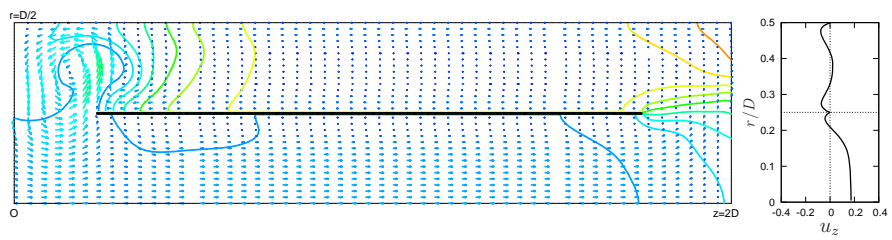

(c) $t-t_{s}=T / 2$

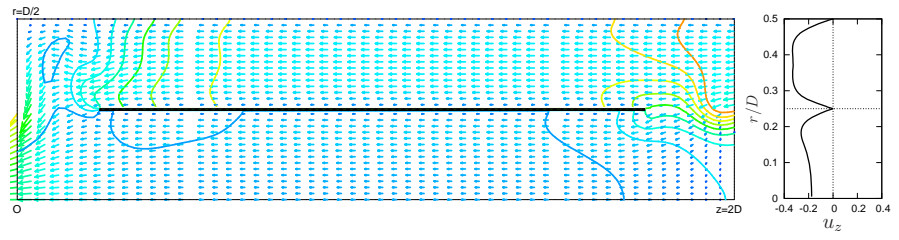

(d) $t-t_{s}=3 T / 4$

Figure 4: Left column: snapshots of the flow and temperature fields for $\chi=1.0$ in a cross-section of $\theta=0$ taken at constant time intervals over one period of oscillation after reaching quasi-steady state $\left(t_{s}=100 T\right)$. Right column: the radial profiles of the axial velocity $u_{z}$ in a cross-section of $z=D$ in the left figures.

In the following, we look into the flow behaviours for the cases of smaller $\chi$ values. Note that, because we keep the flow rate constant for all the cases (including $\chi=1$ case), the inlet velocity becomes larger with decreasing $\chi$.

In the case of $\chi=0.7$ (Fig. 5), the flows in both conduits are different from the coherent oscillating flow as observed in Fig. 4. In the initial one quarter period, a strong jet is formed in the inner conduit, and then it reaches the right-end wall of the chamber with a little attenuation of the jet core. Although the time-averaged net flow rate is zero over one period, larger magnitudes of the velocity vectors are observed in the annulus at $t-t_{s}=3 T / 4$ than those at $t-t_{s}=T / 4$. This asymmetry in velocity magnitudes at the two moments $\left(t-t_{s}=T / 4\right.$ and $\left.3 T / 4\right)$ suggests that the average flow in the annular conduit is in $-z$ direction. On the other hand, the average flow in the inner conduit is in $+z$ direction from the four snapshots and velocity profiles; the out-phase motion of the fluid in the inner and outer conduits at $t-t_{s}=3 T / 4$ is the major 


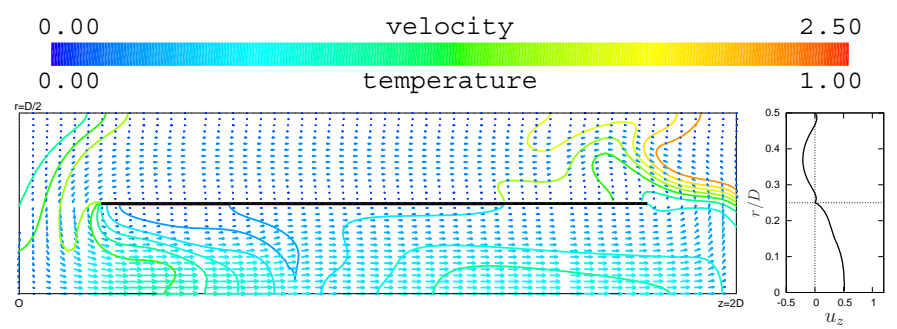

(a) $t-t_{s}=0$

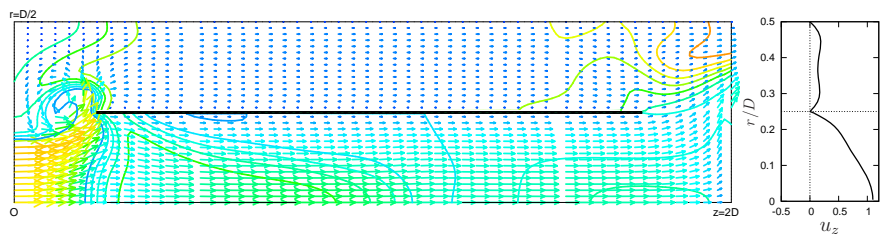

(b) $t-t_{s}=T / 4$
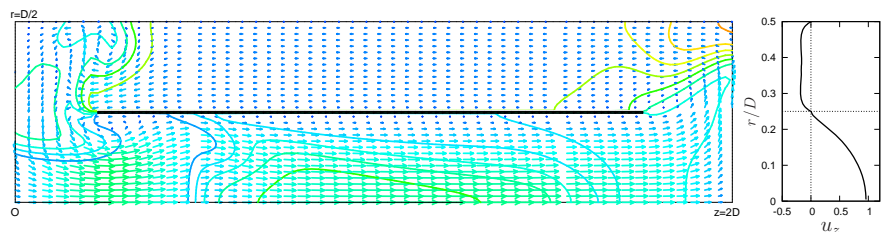

(c) $t-t_{s}=T / 2$

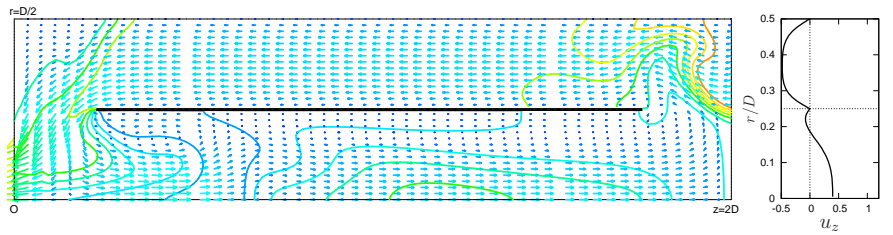

(d) $t-t_{s}=3 T / 4$

Figure 5: Left column: snapshots of the flow and temperature fields for $\chi=0.7$ in a cross-section of $\theta=0$ taken at constant time intervals over one period of oscillation after reaching quasi-steady state $\left(t_{s}=100 T\right)$. Right column: the radial profiles of the axial velocity $u_{z}$ in a cross-section of $z=D$ in the left figures.

difference from the case of $\chi=1$. The overall flow in this coaxial pipe is described as a reciprocal flow superposed on a circulating flow component in the counter-clockwise direction (in this cross-section). The non-zero average flow rates in the respective conduits induced by this circulating component characterises the flow in this coaxial pipe at a value of $\chi$ smaller than unity. Hereafter, the counter-clockwise circulating flow component is referred to as unidirectional current.

The unidirectional current is even pronounced in the case of $\chi=0.5$ (as well as the reciprocal component), as shown in Fig. 6. At $t-t_{s}=T / 4$, a strong shear layer behind the jet front interferes with the left edge of the inner wall, making a pair of vortices: the counter-clockwise vortex works to strengthen the flow from the annulus to the inner tube, generating circulating component (towards the hot end through the inner conduit). On the other hand, the clockwise vortex moves towards the left-top corner (Fig. 6(c)) and, by the time of the outgoing phase at the left-end (Fig. 6(d)), the induced flow by the clockwise vortex assists the 


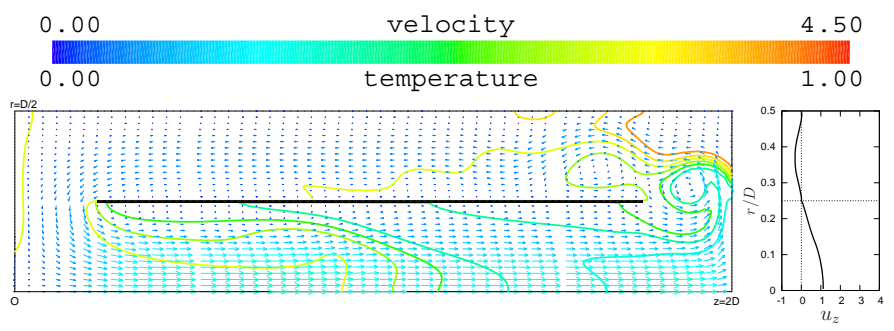

(a) $t-t_{s}=0$

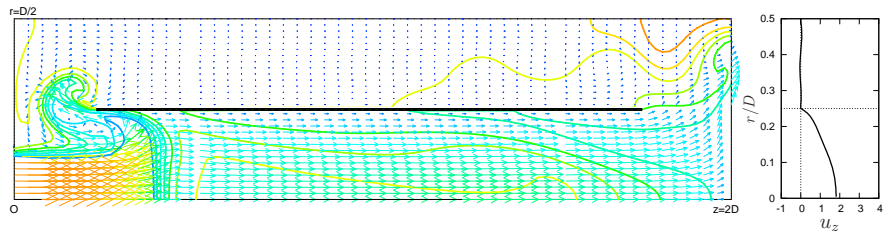

(b) $t-t_{s}=T / 4$
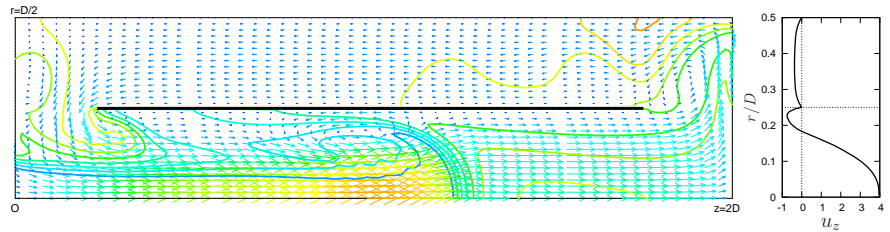

(c) $t-t_{s}=T / 2$

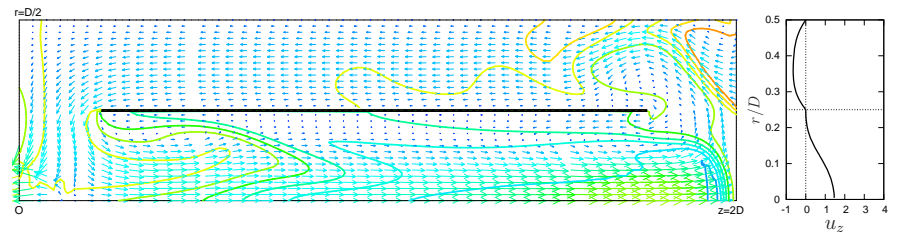

(d) $t-t_{s}=3 T / 4$

Figure 6: Left column: snapshots of the flow and temperature fields for $\chi=0.5$ in a cross-section of $\theta=0$ taken at constant time intervals over one period of oscillation after reaching quasi-steady state $\left(t_{s}=100 T\right)$. Right column: the radial profiles of the axial velocity $u_{z}$ in a cross-section of $z=D$ in the left figures.

returning flow to direct smoothly toward the left exit. Also note that a strong roll-up behind the jet persists downstream in the inner tube.

Looking through the radial profiles of $u_{z}$ in the mid cross-sections (the right columns in Figs $4 \sim 6$ ), the figures clearly show that the average flow rate in the inner and outer conduits are disproportionate in the positive and negative $z$ directions, respectively, with decreasing the $\chi$ values.

\subsection{Flow rate of the circulating component}

Figure 7 shows the development of the flow rate $Q_{F}$ at $z=D$ cross-section for the three cases of $\chi$. From the figures, the flow reaches quasi-steady state by $t=5 T$ for those cases. The flow rate for each case fluctuates around a non-zero value (represented by dashed lines), which corresponds to the average flow 


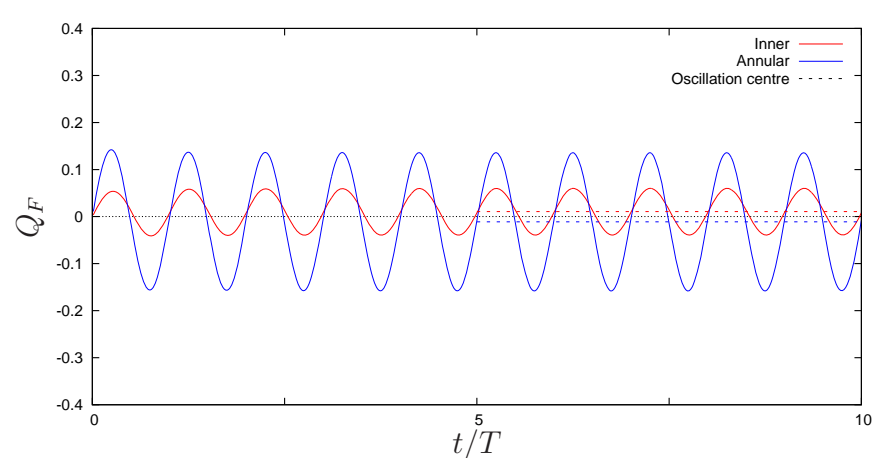

(a) $\chi=1.0$

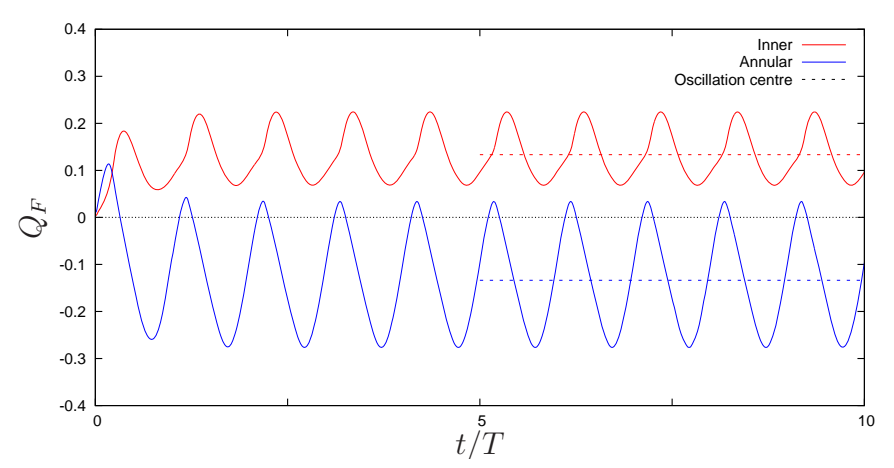

(c) $\chi=0.5$

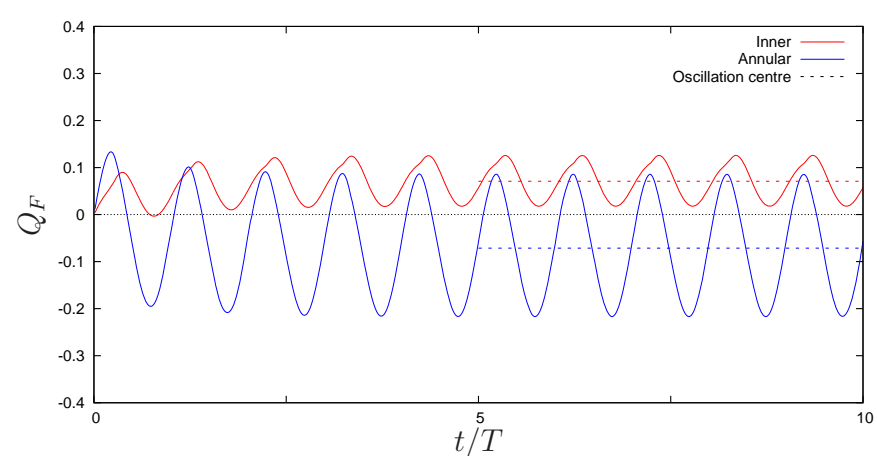

(b) $\chi=0.7$

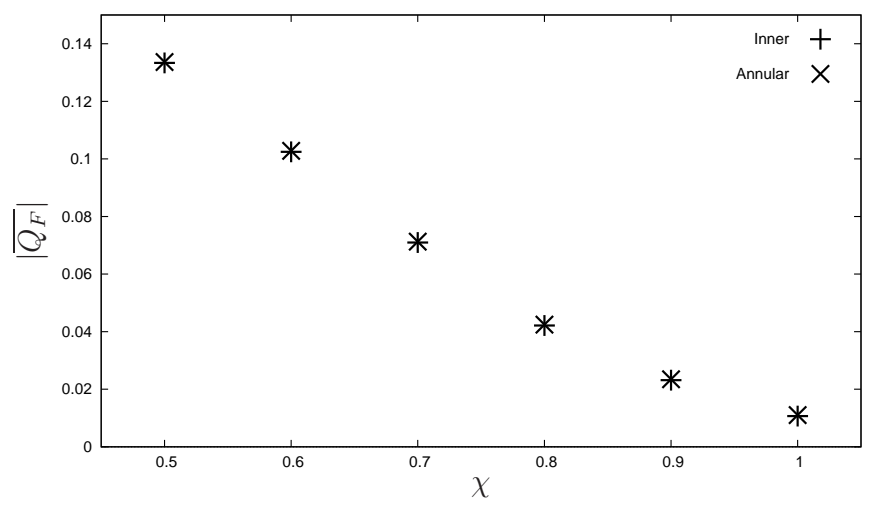

(d)

Figure 7: Flow rate for the cases of different $\chi$ values. (a)-(c): time development of the flow rate at $z=D$ in the inner and outer conduits. The dashed lines show the oscillation centre identified after reaching quasisteady state. (d): comparison of the absolute values of the time-averaged flow rate (taken for $t \geq 5 T$ at $z=D$ cross-section) for different $\chi$.

rate of the unidirectionally circulating component. Taking the average in the time range shown by the dashed lines, the trend of the average flow rate on $\chi$ is summarised in Fig. 7(d). The figure shows that the smaller inlet diameter (smaller $\chi$ value) causes larger magnitude of the average flow rate; for example, the case of $\chi=0.5$ exhibits 12.5 times larger magnitude of the flow rate than the case of $\chi=1.0$.

Figure 8 compares the superficial axial velocity in $z=D$ cross-section over one period of oscillation. The synchronised variation of the velocities in both conduits at $\chi=1.0$ departs as $\chi$ is decreased, and the phase difference enhances accordingly; eventually at $\chi=0.5$ the annulus velocity is about $\pi / 3$ lagged behind the inner velocity. In the inflow phase of the cold-reservoir side $\left(0 \leq t-t_{s} \leq T / 2\right)$, less momentum goes into the annulus at smaller $\chi$, and, at $\chi=0.5$ nearly full-time negative momentum flow is observed in the annulus. In the outflow phase of the cold-reservoir side $\left(T / 2 \leq t-t_{s} \leq T\right)$, the superficial velocity in the inner conduit increases with decreasing $\chi$, and at $\chi=0.7$, no net momentum is transferred towards the cold reservoir through the inner tube. The result reflects the dominant effect of the fluid inertia, causing a larger amount of entrainment from the annulus to the inner tube for smaller $\chi$ cases. A similar role of the fluid inertia has been reported in the bifurcated tube systems [10] for blocking and regulating the flow depending 


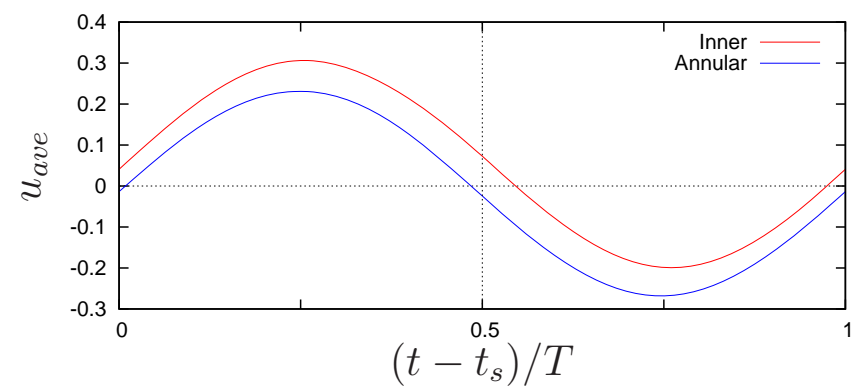

(a) $\chi=1.0$

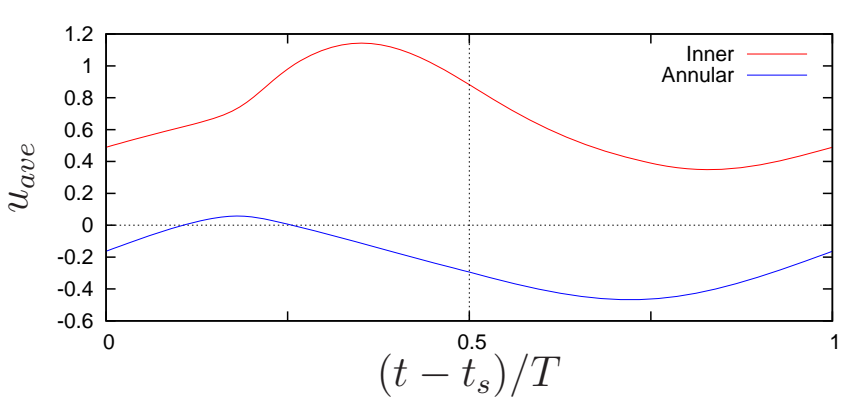

(c) $\chi=0.5$

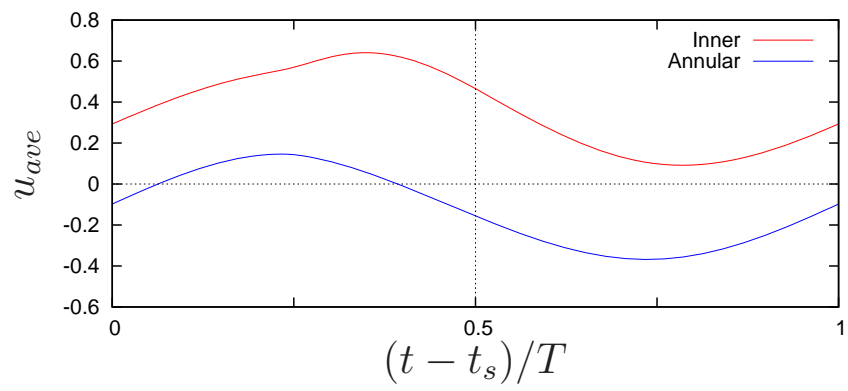

(b) $\chi=0.7$

Figure 8: Time evolution of the flow rate averaged over the cross-section at $z=D$. For $\chi=0.5$ case, the phase of the annulus velocity is lagged by about $\pi / 3$ in comparison to that of the inner velocity.

on the oscillation phases, and the present disproportional fluid inertia in the inflow and outflow phases is the primary cause of the characteristic unidirectional current.

\subsection{Development of vorticity in the cold-reservoir side}

We look at the development of the vorticity in the region ABCD (see Fig. 2). Ishii et al. [11] mentioned that the vortex developed in this region during the inflow phase is closely related to the strength of the unidirectional flow, by changing the oscillating frequency under the condition of fixed diameter ratio $\chi=$ 1.0. The present study is suited for investigating the effect of the inlet vortex as the intensity of the inlet shear layer is controlled by changing the diameter ratio $\chi$ with keeping the inflow rate the same. To see the evolution of vorticity in the region $\mathrm{ABCD}$ (Fig. 2), circulation $\Gamma$ is evaluated in the $r-z$ cross-section:

$$
\Gamma=\oint_{\mathrm{ABCD}} \boldsymbol{u} \cdot \mathrm{d} \boldsymbol{x}
$$

A positive value of $\Gamma$ suggests the presence of a counter-clockwise vortex around $\theta$ axis or shear layer of the same sign.

Figure 9 shows the time developments of the circulations for different $\chi$ values over one period of oscillation after reaching quasi-steady state. Looking at time $t-t_{s}=T / 2$ (the switching moment from the inflow to the outflow phases), the positive $\Gamma$ values for $\chi=1.0 \sim 0.8$ suggest counter-clockwise 


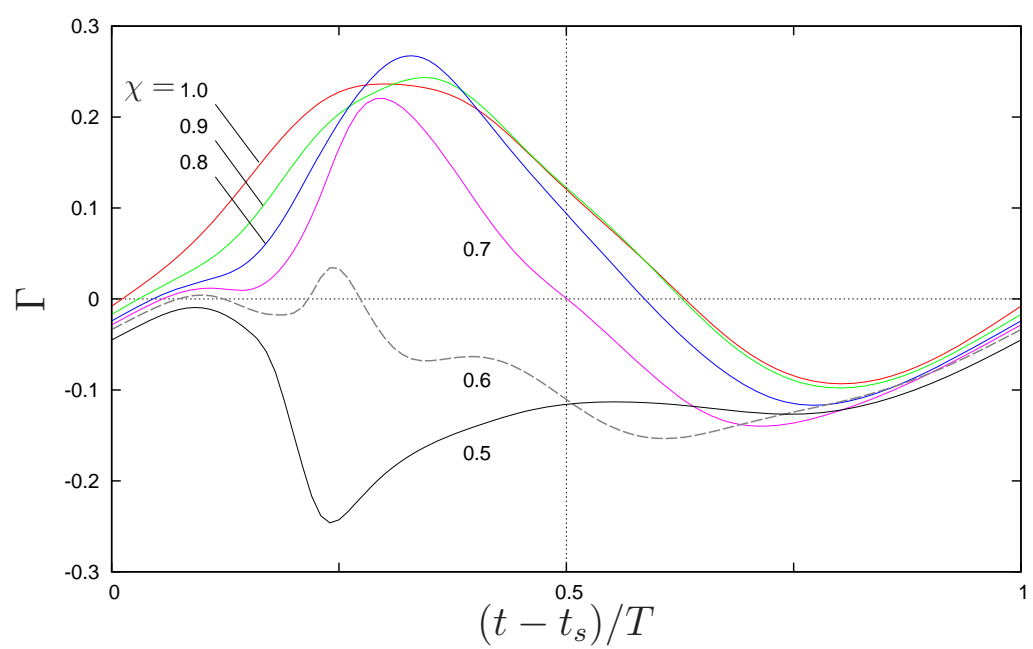

Figure 9: Time variation of the circulation $\Gamma$ (along the path $A B C D$ in Fig. 2) over one period of oscillation for different $\chi$ cases after reaching steady state $\left(t_{s}=100 T\right)$.

vortices as typically observed in Fig. 4(b). These vortices work to decelerate the outflow through the left circular opening (Fig 4(c),(d)). On the other hand, for the case $\chi=0.6$ and 0.5 , negative $\Gamma$ values at $t-t_{s}=T / 2$ suggest a vortex in the clockwise direction, making a smooth out-going flow from the outer conduit through the circular opening at the left end. Particularly for the case of $\chi=0.5$, the circulation is negative throughout the period, and the vortex in the top-left corner of Fig. 6(c) (as well as the shear layer driven by the loop-back flow at the left edge of the inner wall) is responsible for this. The change in vortical structure takes place around $\chi=0.7$ where the average vorticity in one period is approximately zero, and the above results indicate the importance of the development of the negative vortices for smooth outflow and strengthening the unidirectional-circulating flow. The similar trend of the zero-averaged circulation is also observed around $\chi=0.7$ for the cases of different frequencies, $f=0.4$ and 0.6.

\section{Heat-transfer in the oscillation-controlled coaxial pipe}

In this section, we focus on the heat transport characteristics based on the knowledge of the flow evolution in the previous section.

\subsection{Amount of transported heat per input work}

Average transported heat $\overline{Q_{H}}$ is evaluated as follows:

$$
\overline{Q_{H}}=\frac{1}{T} \int_{t}^{t+T} \int_{0}^{2 \pi} \int_{0}^{D / 2}\left(\operatorname{RePr} u_{z} \Theta-\frac{\partial \Theta}{\partial z}\right)_{z=D} r \mathrm{~d} r \mathrm{~d} \theta \mathrm{d} t .
$$




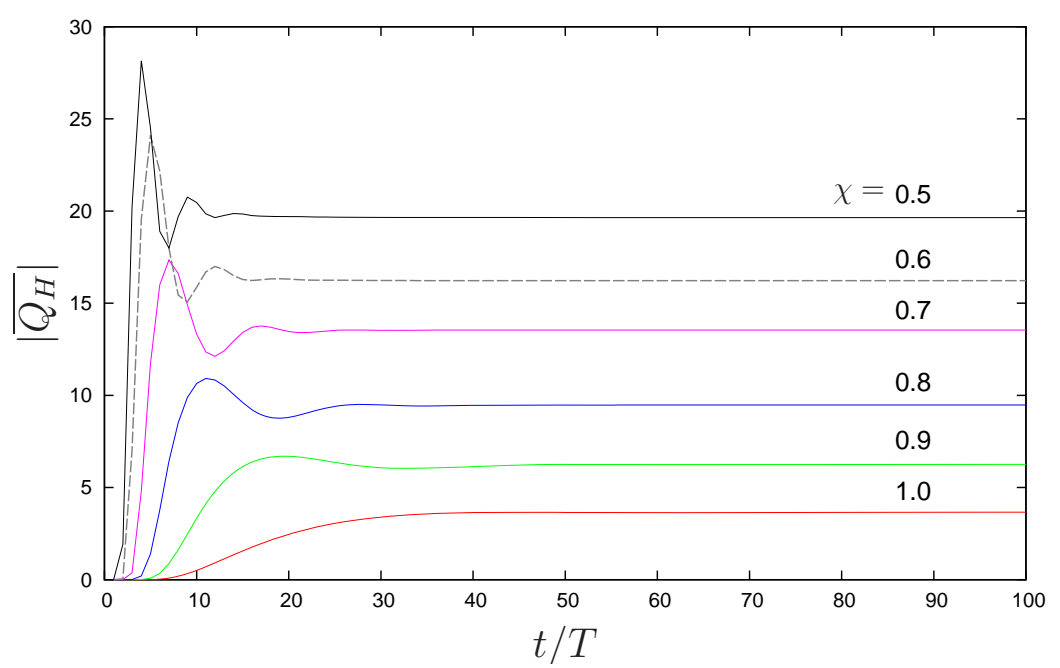

Figure 10: Time variation of the average heat transport rate over one period for the case with the thermallyinsulated inner wall.

Figure 10 shows the time-development of $\overline{Q_{H}}$. Since $z$ direction is taken from the low-temperature region to the hot-temperature region, the amount of transported heat typically shows a negative value. In the figure, the absolute value of $\overline{Q_{H}}$ is plotted. From the figure, $\left|\overline{Q_{H}}\right|$ takes steady values after long time, ranging between about 4 and 20 in the decreasing order of $\chi$. Our preliminary study of diffusion-only case (giving no oscillation of the fluid at both ends) shows approximately $\left|\overline{Q_{H}}\right|=0.34$, which indicates the effectiveness of the convective heat transport in the present device. Figure 11 plots the steady amount of transported heat $\overline{Q_{H}}$ as a function of $\chi$ with a symbol of filled circle. The transported heat in steady state is approximately inversely-proportional to $\chi$, which corresponds to the increase in the flow rate of the unidirectional current by decreasing $\chi($ Fig.7(d)).

However, by decreasing the diameter ratio $\chi$ with keeping the same inflow rate, the input energy to drive the flow into the coaxial-structured chamber would increase. Average mechanical work $\bar{W}$ to drive the fluid taken over one period of oscillation is calculated as:

$$
\bar{W}=\frac{1}{T} \int_{t}^{t+T} \int \boldsymbol{u} \cdot(\nabla \cdot(-p \boldsymbol{I}+\boldsymbol{\tau})) r \mathrm{~d} r \mathrm{~d} \theta \mathrm{d} z \mathrm{~d} t,
$$

where $I$ is the identity tensor and $\tau$ is viscous stress tensor. Note that this mechanical work does not consider the energy loss of the fluid due to the radial constriction from/to the reservoirs of large volumes. Then, heat transport coefficient $\epsilon$ is defined as the transported heat per unit input energy:

$$
\epsilon=\operatorname{Tr} \frac{\left|\overline{Q_{H}}\right|}{\bar{W}},
$$

where $\operatorname{Tr}$ is the following non-dimensional number:

$$
\operatorname{Tr}=\frac{\kappa \Delta \Theta}{\rho U^{3} D}
$$




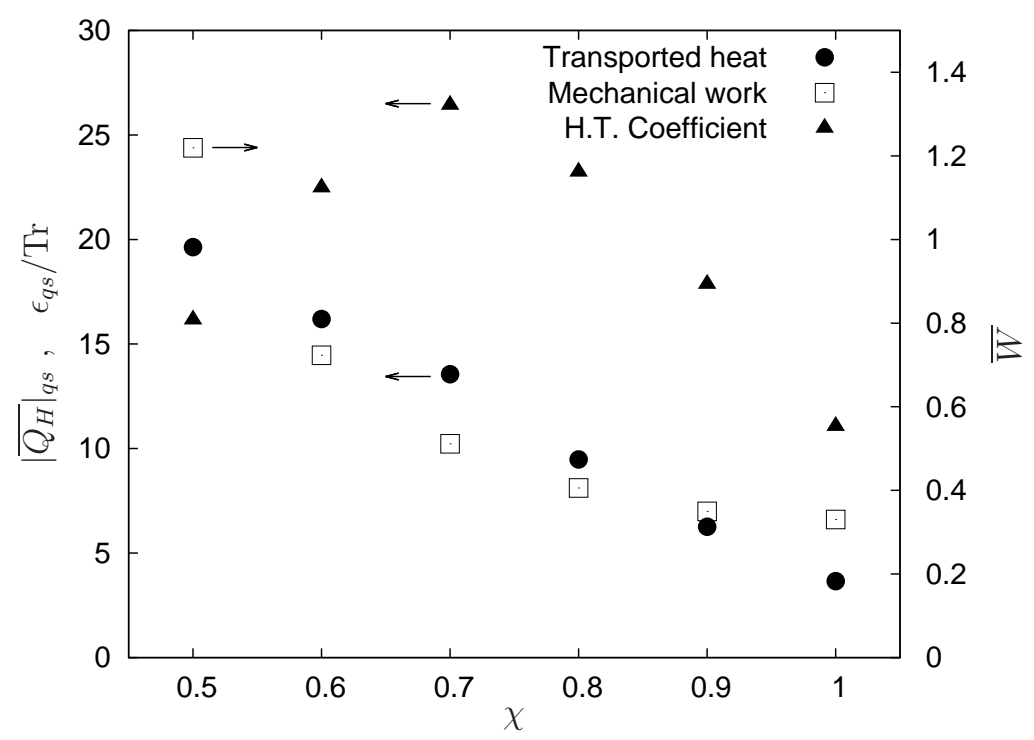

Figure 11: Characteristics of heat transport and mechanical work after reaching steady state plotted against the diameter ratio $\chi$.

This non-dimensional number, the ratio of the conductive heat flux to the mechanical work by pressure, represents the effectiveness of the heat transport. Meanwhile, Tr is also represented with other variables as follows:

$$
\operatorname{Tr}=\frac{1}{\operatorname{Re} \operatorname{Pr}} \frac{\rho c \Delta \Theta}{\rho U^{2}}=\frac{1}{\operatorname{Re}^{2} \operatorname{Pr}} \frac{\rho c \Delta \Theta}{\mu U / D},
$$

where $c$ is the specific heat and $\mu$ is the viscosity of the working fluid. Those are interpreted as the amount of heat exchanged by the pressure difference and against the shear stress, respectively. Therefore, both notations of the heat transport effectiveness parameter represent the characteristics of the present device.

The tendency of $\bar{W}$ (in quasi-steady state) with respect to $\chi$ plotted in Fig. 11 confirms that the driving power increases as decreasing the inlet diameter. The heat transport coefficients $\epsilon / \operatorname{Tr}$ against $\chi$ is also plotted in Fig. 11 with a triangular symbol, showing that the heat transport rate takes the local maximum value at around $\chi=0.7$. The result suggests the presence of the optimal geometric condition for the efficient heat transport, and in the present problem set-up, the optimal diameter ratio exists around $\chi=0.7$. The heat transport rate is closely related to the flow structure; while we saw in Fig.9 that the development of the negative vortices in the region $\mathrm{ABCD}$ facilitates the smooth outflow and a spontaneous unidirectional flow, the above result of the optimal performance at around $\chi=0.7$ suggests that the zero-averaged vorticity in one period is of significance in terms of the heat transfer efficiency.

The similar trend of local maxima of $\epsilon$ at around the same $\chi$ are observed for the other cases of oscillation frequencies between 0.4 and 0.6 . However, the largest decrease in $\epsilon$ of about $18 \%$ is found at $\chi=0.7$ for the 


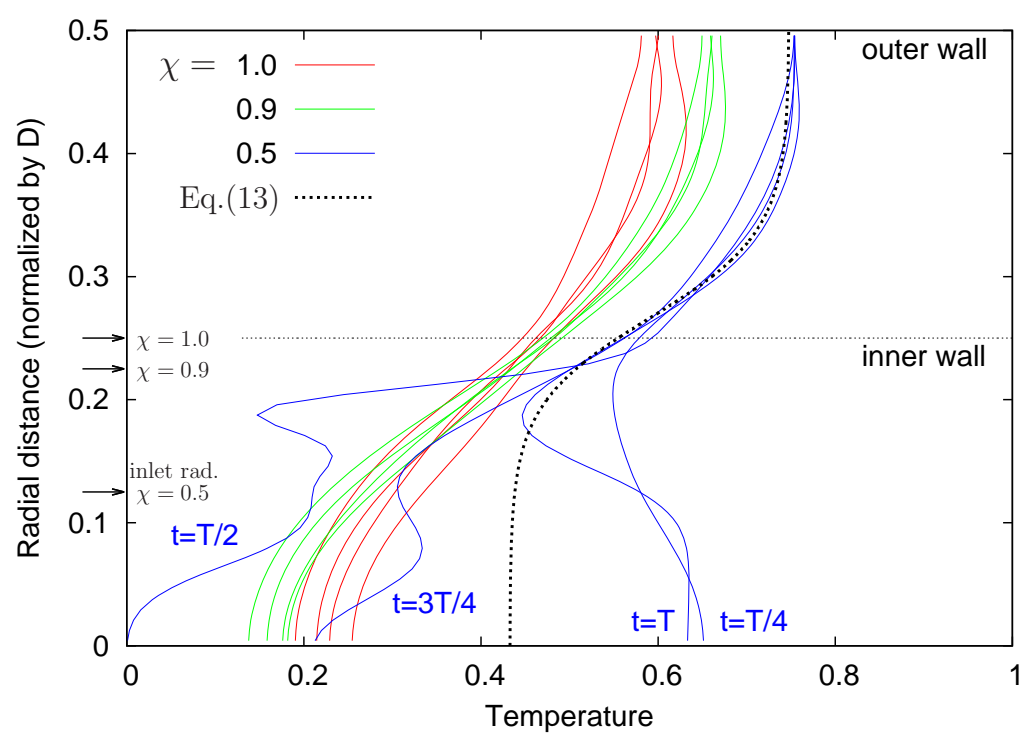

Figure 12: Instantaneous radial profiles of temperature in $z=D$ cross-section for $\chi=1.0,0.9$ and 0.5 cases at constant intervals. The profiles of $\chi=0.5$ is fitted with a tangent-hyperbolic function.

anti-synchronised case of $f=0.6$, and at the frequencies in the above range, slight decrease and increase in the transported heat and the mechanical work, respectively, result in non-negligible reduction in the values of $\epsilon$ in the overall range of $\chi$.

\subsection{Effect of the conductive inner tube wall}

Thus far, the inner tube is assumed to be thermally-insulated; no heat exchange between the fluids in the inner and outer conduits across the inner wall. Insulated wall is an idealised situation, and, with the heat conduction across the inner wall, the heat transport coefficient is supposed to decrease due to the heat loss from the annulus to the inner side. Effect of the conductive inner wall gives further deep understandings on the roles of the unidirectional current and the flow structure.

Here, for replicating the fully-conductive (but no-permeable) wall, the Neumann condition for the temperature on the inner wall is eliminated and solved with the same limiting values of the wall temperature from both sides of the inner tube wall.

Figure 12 compares the instantaneous radial temperature profiles for three different diameter ratios obtained at constant intervals over one period of oscillation after reaching quasi-steady state. For the cases of weak unidirectional-circulating component $(\chi=1.0$ and 0.9$)$, the profiles are similar to each other within each $\chi$ case, reflecting a smooth and regular pulsating flow. For the case of $\chi=0.5$, on the other hand, the temperatures in the inner conduit are strongly disturbed due to the passage of a strong jet, while the outer conduit is relatively calm around a high temperature of $\Theta=0.7$. 


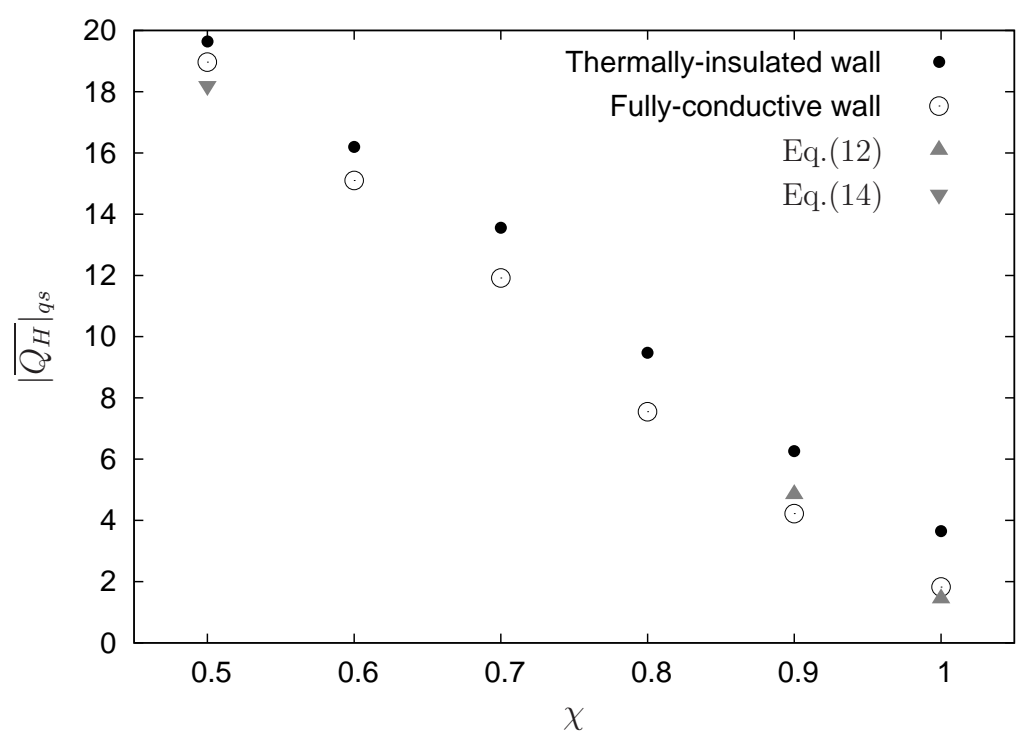

Figure 13: Averaged transported heat over one period of oscillation against $\chi$ for the cases of the conductive wall (open symbol). Also plotted are the transported heat for the case of the insulated wall (filled circular symbol; representing the identical data to those in Fig. 11) and modelled heat transfer rate (triangular symbols).

For the transported heat with the conductive inner wall, a similar time-development of $\left|\overline{Q_{H}}\right|$ to Fig. 10 is observed with a little decrease in magnitude for each $\chi$ case. The steady amounts of the transported heat (calculated by Eq.(7)) are plotted in Fig. 13 with open symbol and compared to the case with the insulated walls (identically shown in Fig. 11) with filled circular symbol. The largest decrease is found at $\chi=1.0$ showing only $51 \%$ of the insulated case is transported, whereas the case of $\chi=0.5$ is little affected with only a $4 \%$ decrease. This decrease trend of $\left|\overline{Q_{H}}\right|$ is opposite to that of the strength of the unidirectional component (Fig.7(d)). A simplified model of convective heat transfer explains this. For $\chi=1.0$ and 0.9 , assuming that the radial temperature profile is linearly correlated with $r$ (i.e., $\left.\Theta=\left.\frac{\partial \Theta}{\partial r}\right|_{R_{i}}\left(r-R_{i}\right)+\left.\Theta\right|_{R_{i}}\right)$ and that the heat transfer is mainly resulted from the convection by the unidirectional flow component, we readily obtain the following average heat transfer rate in the cross-section of $z=D$ :

$$
\overline{Q_{H}}=-\left.\frac{2}{9} \operatorname{Re} \operatorname{Pr} \overline{Q_{F}} D \frac{\overline{\partial \Theta}}{\partial r}\right|_{R_{i}} .
$$

Here, the average temperature gradient at the inner tube $\left(R_{i}=D / 4\right)$ are obtained from Fig. 12 and the flow rate $\overline{Q_{F}}$ in Fig. 7(d) is substituted. The obtained $\left|\overline{Q_{H}}\right|_{q s}$ are plotted in Fig. 13 with upper triangular symbol, showing reasonable agreement.

On the other hand, for $\chi=0.5$ case, a strong vortex travels in the inner conduit in the wake of the discharged jet towards the hot end, as observed in Fig. 6(c). The temperature distribution in this buffer region (between the wall and the jet column) would not contribute to the net convective heat transfer very 
much, while the jet core is strongly responsible for. Here we assume a tangent-hyperbolic-type radial temperature distribution of the following form:

$$
\Theta_{\chi=0.5}=c_{1}+c_{2}\left(1+\tanh \left(c_{3}\left(r-c_{4}\right)\right)\right) \quad\left(c_{i}: \text { Const. }\right)
$$

as shown in Fig. 12 with a dotted line, and the only contribution by the jet core region $\left(0 \leq r \leq \chi R_{i}\right)$ is taken into account for the heat transport through the inner conduit to obtain the following average heat transport rate:

$$
\overline{Q_{H}}=2 \pi \operatorname{Re} \operatorname{Pr}\left[\int_{0}^{\chi R_{i}} \frac{\left|\overline{Q_{F}}\right|}{\pi\left(\chi R_{i}\right)^{2}} \Theta_{\chi=0.5} r \mathrm{~d} r-\int_{R_{i}}^{R_{o}} \frac{16\left|\overline{Q_{F}}\right|}{3 \pi D^{2}} \Theta_{\chi=0.5} r \mathrm{~d} r\right] .
$$

The result is plotted in Fig. 13 with inverted-triangular symbol, and the acceptable agreement to the numerical result indicates that the region between the inner wall and jet core do not play major contribution to the heat transport for the case of $\chi=0.5$.

Despite the above crude approximations, the results suggest that, after the development of the temperature distribution resulted from the heat conduction across the inner tube, the heat transfer is modelled with the advection by the unidirectional-circulating component. Although large temperature difference exists between the inner and outer conduits for the smaller $\chi$ cases, the heat loss across the conductive inner wall is limited and possibly circumvented by allowing to emerge a strong jet for separating the hot fluid in the outer conduit from the cold fluid in the jet core. Due to the less-active transport effect by this buffer region, the heat transfer performance in quasi-steady state is less affected by the heat loss across the conductive inner wall. This flow characteristics is one of the advantages of the oscillation-controlled coaxial pipe.

\section{Conclusions}

Flow structure and heat transport characteristics of an oscillation-controlled heat-transport coaxial pipe were numerically studied. Cold and hot fluids in the reservoirs are discharged by anti-phase reciprocal waves through a circular and annular openings at the respective ends of the chamber.

Due to the double-tube structure of the chamber, a unidirectional-circulating flow going through inner and outer conduits was observed to develop spontaneously in a range of diameter ratios (of the circular opening to the inner tube diameter) between 1.0 and 0.5 . Particularly at the small diameter ratios (with keeping the inflow rate the same), development of a strong jet column and the passage of the jet into the inner tube enhanced the entrainment of the fluid from the annulus to the inner tube, resulting in a strong unidirectional current. The flow rates of the unidirectional currents were inversely-correlated to the diameter 
ratio. Also, in the cases of the small diameter ratios $(0.6$ and 0.5$)$, the vortex-induced flow was observed to facilitate the annulus fluid to return to the cold reservoir in the outgoing phase at the cold end, whereas approximately zero-averaged vorticity developed in one period for the case of the diameter ratio 0.7 .

With an ideally insulated inner wall, the amount of transported heat increased with the flow rate of the unidirectional current. In the cases employing a conductive inner wall, the heat loss due to the heat conduction across the inner wall was found to be suppressed with decreasing the diameter ratio, due to the less-active transport effect in the region between the conductive wall and the jet core (developing away from the inner wall). This is one of the advantages of this coaxial heat-transport pipe for practical applications.

However, the study shows that those advantages have to be judged together with the mechanical work for driving the fluid in and out of the chamber. Indeed, the mechanical work also increased as the diameter ratio is decreased; the increase is even at a higher rate at the smaller diameter ratios, and the highest heat transfer coefficient (i.e. the transported heat per unit mechanical work) was found to exist around the diameter ratio of 0.7 in the present study. The results suggest that, while generating the strong jet and vortices near the circular opening effectively works for strengthening the unidirectional component (and, in turn, the amount of the transported heat), the zero-averaged vorticity (i.e. not letting stay a standing vortex) is of significance for improving the heat transfer performance.

The present study showed the importance of the cold-side opening diameter for determining and predicting the performance of the oscillation-controlled coaxial heat-transport pipe, and revealed that the unidirectional current and vortical structures interplay for the transport phenomena of the heat and mass.

\section{References}

[1] Kurzweg, U.H. and Zhao, L.D. "Heat transfer by high-frequency oscillations: A new hydrodynamic technique for achieving large effective thermal conductivities", Physics of Fluids, Vol.27, No.11 (1984), pp.2624-2627.

[2] Watson, E.J., "Diffusion in oscillatory pipe flow", Journal of Fluid Mechanics, Vol.133 (1983), pp.233-244.

[3] Jaeger, M.J. and Kurzweg, U.H., "Determination of the longitudinal dispersion coefficient in flows subjected to high-frequency oscillations", Physics of Fluids, Vol.26 (1983), pp.1380-1382. 
[4] Joshi, C.H., Kamm, R.D., Drazen, J.M. and Slutsley, A.S., "An experimental study of gas exchange in laminar oscillatory flows", Journal of Fluid Mechanics, Vol.133 (1983), pp.245-254.

[5] Kurzweg, U.H., Howell, G. and Jaeger, M.J., "Enhanced dispersion in oscillatory flows", Physics of Fluids, Vol.27 (1984), pp.1046-1048.

[6] Schmidt-Nielsen, K., Animal Physiology: Adaption and Environment (Fifth edition), Cambridge University Press (1997).

[7] Farmer, C.G. and Sanders, K. "Unidirectional Airflow in the Lungs of Alligators", Science, Vol.327 (2010) pp.338-340

[8] Schachner, E.R., Cieri, R.L., Butler, J.P. and Farmer, C.G. "Unidirectional pulmonary airflow patterns in the savannah monitor lizard", Nature, Vol.506 (2014) pp.367-370

[9] Wang, N., Banzett, R.B., Butler, J.P. and Fredberg, J.J. "Bird lung models show that convective inertia effects inspiratory aerodynamic valving", Respiration Physiology, Vol.73 (1988) pp.111-124.

[10] Sakai, E., Watanabe, T. and Himeno, T., "Generation mechanism of unidirectional flow in oscillatory flow of avian lung", Journal of Flow Visualization and Image Processing, Vol.13 Issue 2 (2006) pp.113-132.

[11] Ishii, Y., Zhang, P., Kawai, R., Hishida, M. and Tanaka, G., "Heat transportation by an oscillatory flow in a double tube", Proc. JSME Thermal Engineering Conference (2005), pp445-446.

[12] Hishida, M., Mitsuno, R., Zhang, X. and Tanaka, G. "Heat transportation by oscillatory flow in a new type of heat transportation pipe", International Journal of Heat and Mass Transfer, Vol.52 (2009), pp.5634-5642.

[13] Kaviany, M., "Performance of a heat exchanger based on enhanced heat diffusion in fluids by oscillation: Analysis", Journal of Heat Transfer, Vol.71 (1975), pp.513-527.

[14] Inaba, T., Tahara, M. and Saitoh, K., "Longitudinal heat transfer in oscillatory flows in pipe bundles of various cross sections", JSME International Journal Series B, Vol.43(3) (2000), pp.460-467.

[15] Nishio, S., Shi, X.-H. and Zhang, W.-M., "Oscillation-induced heat transport: Heat transport characteristics along liquid-columns of oscillation-controlled heat transport tubes", International Journal of Heat and Mass Transfer, Vol.38 Issue 13 (1995), pp.2457-2470. 
[16] Amsden,A.A. and Harlow,F.H. "A simplified MAC technique for incompressible fluid flow calculations”, Journal of Computational Physics, Vol.6 (1970), pp.322-325

[17] Takeuchi, S., Miyake, Y., Kajishima, T. and Aoki, S., "Direct numerical simulation of turbulent round jet”, Transaction of JSME Ser. B, Vol.65-640 (1999), pp.3918-3925

[18] Takeuchi, S., Miyake, Y. and Kajishima, T., "Secondary instability of round jets by the global instability analysis", Transaction of JSME Ser. B, Vol.68-665 (2002), pp.30-37 


\section{Nomenclature}

$\begin{array}{ll}C_{J}, C_{Y} & \text { Coefficient } \\ D & \text { Diameter } \\ \boldsymbol{I} & \text { Identity tensor } \\ J_{0} & \text { 0-th order Bessel function of the 1st kind } \\ L & \text { Reference length } \\ N_{j} & \text { Number of grid points in } j \text {-direction } \\ \operatorname{Pr} & \text { Prandtl number } \\ Q_{F} & \text { Flow rate } \\ Q_{H} & \text { Transported heat per unit time } \\ \operatorname{Re} & \text { Reynolds number } \\ R_{i} & \text { Inner radius } \\ R_{o} & \text { Outer radius } \\ T & \text { Period of oscillation } \\ \text { Tr } & \text { Heat transport effectiveness parameter } \\ U & \text { Maximum centreline velocity of jet } \\ U_{m L} & \text { Mean velocity from the left end } \\ U_{m R} & \text { Mean velocity from the right end } \\ V & \text { Reference velocity } \\ W & \text { Mechanical work } \\ Y_{0} & \text { 0-th order Bessel function of the 2nd kind } \\ c & \text { Specific heat } \\ \ell & \text { Subdomain length } \\ f & \text { Frequency } \\ i & \text { Imaginary unit } \\ m, n & \text { Arbitrary integer } \\ p & \text { Pressure } \\ r & \text { Radial coordinate } \\ t & \text { Time } \\ t_{d}, t_{s} & \text { Base point in time } \\ u & \text { Velocity vector } \\ u_{j} & \text { Velocity component in } j \text {-direction } \\ z & \text { Axial coordinate } \\ & \end{array}$

\section{Greek symbols}

$\Xi \quad$ Differential operator

$\Delta \Theta \quad$ Characteristic temperature difference

$\Gamma \quad$ Circulation

$\Lambda \quad$ Differential operator

$\Theta \quad$ Temperature

$\alpha \quad \sqrt{i^{3} \omega / \nu}$

$\epsilon \quad$ Heat transport coefficient

$\theta \quad$ Azimuthal coordinate

$\kappa \quad$ Thermal conductivity

$\mu \quad$ Viscous coefficient

$\nu \quad$ Kinematic viscosity

$\rho \quad$ Density

$\tau \quad$ Viscous stress tensor

$\phi \quad$ Phase angle

$\chi \quad$ Diameter ratio

$\omega \quad$ Angular frequency

\section{Subscripts and superscripts}

$\begin{array}{ll}L & \text { Left end of the chamber } \\ R & \text { Right end of the chamber } \\ \text { ann } & \text { Annular conduit } \\ \text { ave } & \text { Average } \\ \text { circ } & \text { Circular conduit } \\ \text { qs } & \text { Quasi-steady } \\ \text { overline } & \text { Time average }\end{array}$

